\title{
Translation-invariant bipolarons and the problem of high-temperature superconductivity.
}

\author{
V.D. Lakhno ${ }^{\mathrm{a}, *}$ \\ ${ }^{a}$ Institute of Mathematical Problems of Biology, Russian Academy of Sciences, Pushchino, Moscow Region, 142290, Russia
}

\begin{abstract}
- It is shown that the bipolaron ground state is described by a delocalized wave function. For a two-parameter wave function, the lowest variation estimate of the ground state energy in the strong coupling limit is found to be $E=-0,414125 \alpha^{2}$. This is much lower than that derived with the use of the localized bipolaron wave function. The results obtained testify to the possibility of a bipolaron mechanism of high-temperature superconductivity.
\end{abstract}

'Keywords: A. Superconductors, C. Delocalized Bipolarons, D. Froehlich Hamiltonian, D. Lee, Low and Pines transformation

\section{Introduction}

The problem of possible arising of high-temperature superconductivity (HTSC) and explanation of this phenomenon by the existence of bipolaron states was studied in a number of papers (see reviews [1, 2]). There the occurrence of HTSC is treated via the mechanism of Bose-condensation of bipolaron gas. The temperature of Bose-condensation $T_{0}=$ $3,31 \hbar^{2} n_{0}^{2 / 3} / k_{B} m_{B P}$ which in this case can be identified with the critical temperature $T_{c}$ of the transition into the superconducting state at $m_{B P}^{*} \approx 10 m_{0}$ depending on the bipolarons concentration $n_{0}$ varies in a wide range from $T_{0} \approx 3 K$ for $n \approx 10^{18}$ $\mathrm{cm}^{-3}$ to $T_{0} \approx 300 K$ for $n \approx 10^{21} \mathrm{~cm}^{-3}$. In the latter case the bipolarons concentration is so considerable that for bipolaron gas as well as for superconducting pairs, a bipolaron, being a composite formation should no longer conduct itself as an ideal Bose-particle, and at still greater concentrations it should decompose into individual polarons. In this connection the study of conditions at which bipolaron states are stable takes on primary importance. The lowest values of the bipolaron energy were obtained for the electron-phonon coupling constant $\alpha<8$ in [3, 4, 5], and for $\alpha>8$ in [5, 6, 7].

In paper [8] the lowest energy of the bipolaron energy was found for $\alpha>8$. Here our aim is to improve that estimate.

\section{Results and Discussion}

We will proceed from Froehlich Hamiltonian for the bipolaron:

\footnotetext{
${ }^{*}$ Corresponding author

Email address: lak@impb.psn.ru (V.D. Lakhno)
}

$$
\begin{array}{r}
\hat{H}=-\frac{\hbar^{2}}{2 m} \Delta_{r_{1}}-\frac{\hbar^{2}}{2 m} \Delta_{r_{2}} \\
+\sum_{k} \hbar \omega a_{k}^{+} a_{k}+U\left(\left|\vec{r}_{1}-\vec{r}_{2}\right|\right) \\
+\sum_{k}\left(V_{k} e^{i k r_{1}} a_{k}+V_{k} e^{i k r_{2}} a_{k}+\text { h.c. }\right), \\
U\left(\left|\vec{r}_{1}-\vec{r}_{2}\right|\right)=e^{2} / \varepsilon_{\infty}\left|\vec{r}_{1}-\vec{r}_{2}\right|,
\end{array}
$$

where $m$ is the electron effective mass; $r_{1}, r_{2}$ are coordinates of the first and second electron, respectively; $a_{k}^{+}, a_{k}$ are operators of birth and annihilation of phonons with energy $\hbar \omega$,

$$
V_{k}=\frac{e}{k} \sqrt{\frac{2 \pi \hbar \omega}{\tilde{\varepsilon} V}}, \quad \tilde{\varepsilon}^{-1}=\varepsilon_{\infty}^{-1}-\varepsilon_{0}^{-1},
$$

where $e$ is the electron charge, $\varepsilon_{\infty}$ and $\varepsilon_{0}$ are high-frequency and static dielectric permittivities, $V$ is the crystal's volume.

In the center-of-mass system, Hamiltonian (1) takes the form:

$$
\begin{array}{r}
\hat{H}=-\frac{\hbar^{2}}{2 M_{e}} \Delta_{R}-\frac{\hbar^{2}}{2 \mu_{e}} \Delta_{r}+U(|\vec{r}|)+\sum_{k} \hbar \omega a_{k}^{+} a_{k} \\
+\sum_{k} 2 \cos \frac{\vec{k} \vec{r}}{2}\left(V_{k} a_{k} e^{i \vec{k} \vec{R}}+h . c .\right), \\
\vec{R}=\left(\vec{r}_{1}+\vec{r}_{2}\right) / 2, \quad \vec{r}=\left(\vec{r}_{1}-\vec{r}_{2}\right), \\
M_{e}=2 m, \quad \mu_{e}=m / 2 .
\end{array}
$$

In what follows we will use the units in which $\hbar=1, \omega=1$, $M_{e}=1$ (accordingly $\left.\mu_{e}=1 / 4\right)$.

The center-of-mass coordinate $\vec{R}$ can be eliminated from (3) with the use of Heisenberg canonical transformation :

$$
\hat{S}_{1}=\exp \left\{-i \sum_{k} \vec{k} a_{k}^{+} a_{k}\right\} \vec{R}
$$




$$
\begin{array}{r}
\hat{H}=\hat{S}_{1}^{-1} \hat{H} \hat{S}_{1}=-2 \Delta_{r}+U(|\vec{r}|)+\sum_{k} a_{k}^{+} a_{k} \\
+\sum_{k} 2 \cos \frac{\vec{k} \vec{r}}{2}\left(V_{k} a_{k}+V_{k}^{*} a_{k}^{+}\right)+\frac{1}{2}\left(\sum_{k} \vec{k} a_{k}^{+} a_{k}\right)^{2} .
\end{array}
$$

From (5) it follows that the exact solution of the bipolaron problem, determined by the wave function $\Psi(r)$ contains only relative coordinates $r$ and is, therefore, translationally-invariant.

Averaging $\hat{\hat{H}}$ over $\Psi(r)$ we rewrite (5) as Hamiltonian $\hat{\hat{H}}$ :

$$
\begin{array}{r}
\hat{H}=\frac{1}{2}\left(\sum_{k} \vec{k} a_{k}^{+} a_{k}\right)^{2}+\sum_{k} a_{k}^{+} a_{k} \\
+\sum_{k} \bar{V}_{k}\left(a_{k}+a_{k}^{+}\right)+\bar{T}+\bar{U}, \\
\bar{V}_{k}=2 V_{k}\left\langle\Psi\left|\cos \frac{\vec{k} \vec{r}}{2}\right| \Psi\right\rangle, \quad \bar{U}=\langle\Psi|U(r)| \Psi\rangle, \\
\bar{T}=-2\left\langle\Psi\left|\Delta_{r}\right| \Psi\right\rangle .
\end{array}
$$

Lee, Low and Pines canonical transformation [9] of this Hamiltonian:

$$
\hat{S}_{2}=\exp \left\{\sum_{k} f_{k}\left(a_{k}-a_{k}^{+}\right)\right\}
$$

yields:

$$
\begin{array}{r}
\hat{\tilde{H}}=\hat{S}_{2}^{-1} \hat{H} \hat{S}_{2}, \quad \hat{\tilde{H}}=\hat{H}_{0}+\hat{H}_{1}, \\
\hat{H}_{0}=\bar{T}+\bar{U}+2 \sum_{k} \bar{V}_{k} f_{k}+\sum_{k} f_{k}^{2}+\frac{1}{2}\left(\sum_{k} \vec{k} f_{k}\right)^{2} \\
+\sum_{k}\left(1+\frac{k^{2}}{2}+\vec{k} \sum_{k^{\prime}} \overrightarrow{k^{\prime}} f_{k^{\prime}}^{2}\right) a_{k}^{+} a_{k} \\
+\frac{1}{2} \sum_{k, k^{\prime}}\left(\vec{k} \overrightarrow{k^{\prime}}\right) f_{k} f_{k^{\prime}} \\
\cdot\left(a_{k} a_{k^{\prime}}+a_{k}^{+} a_{k^{\prime}}^{+}+a_{k}^{+} a_{k^{\prime}}+a_{k} a_{k^{\prime}}^{+}\right), \\
\hat{H}_{1}=\sum_{k}\left[\bar{V}_{k}+f_{k}\left(1+\frac{k^{2}}{2}+\vec{k} \sum_{k^{\prime}} \overrightarrow{k^{\prime}} f_{k^{\prime}}^{2}\right)\right]\left(a_{k}+a_{k}^{+}\right) \\
+\sum_{k, k^{\prime}}\left(\vec{k} \overrightarrow{k^{\prime}}\right)\left[f_{k^{\prime}} a_{k}^{+} a_{k} a_{k^{\prime}}+f_{k^{\prime}} a_{k}^{+} a_{k^{\prime}}^{+} a_{k}\right] \\
+\frac{1}{2} \sum_{k, k^{\prime}}\left(\vec{k} \overrightarrow{k^{\prime}}\right) a_{k}^{+} a_{k^{\prime}}^{+} a_{k} a_{k^{\prime}} .
\end{array}
$$

The probe $\mathrm{WF} \Psi_{0}$ for minimizing the energy determined by $\hat{\tilde{H}}$ is chosen as:

$$
\begin{aligned}
\Psi_{0}= & \Psi(r) \exp \left\{-i \sum_{k} \vec{k} a_{k}^{+} a_{k} \vec{R}\right\} \\
& \cdot \exp \left\{\sum_{k} f_{k}\left(a_{k}-a_{k}^{+}\right)\right\} \Lambda_{0},
\end{aligned}
$$

where $\Lambda_{0}$ is the eigen wave function of operator (9) [8, 10]. As a result we express the energy $E$ of the bipolaron ground state as:

$$
E=\Delta E+2 \sum_{k} \bar{V}_{k} f_{k}+\sum_{k} f_{k}^{2}+\bar{T}+\bar{U}
$$

where

$$
\begin{array}{r}
\Delta E=\frac{1}{4 \pi^{2}} \int_{0}^{\infty} \frac{k^{4} f_{k}^{2} d k}{(1+Q)}+\frac{1}{12 \pi^{4}} \int_{0}^{\infty} k^{4} p^{4} f_{k}^{2} f_{p}^{2} \\
\frac{\omega_{p}\left(\omega_{k} \omega_{p}+\omega_{k}\left(\omega_{k}+\omega_{p}\right)+1\right)}{\left(\omega_{k}+\omega_{p}\right)^{2}\left(\omega_{p}^{2}-1\right)\left|D_{+}\left(\omega_{p}^{2}\right)\right|^{2}} d p d k, \\
Q=\frac{1}{3 \pi^{2}} \int_{0}^{\infty} \frac{k^{4} f_{k}^{2} \omega_{k}}{\left(\omega_{k}^{2}-1\right)} d k .
\end{array}
$$

Notice that in accordance with [8, 10] the bipolaron wave function is delocalized with respect to $\mathrm{R}$. To get the energy of bipolaron ground state we will use variational approach and assume:

$$
\begin{aligned}
f_{k} & =-\bar{V}_{k} \exp \left(-k^{2} / 2 \mu\right), \\
\Psi(r) & =\left(\frac{2}{\pi l^{2}}\right)^{3 / 4} \exp \left(-\frac{r^{2}}{l^{2}}\right)
\end{aligned}
$$

where $\mu$ and $l$ are variational parameters. Notice, that (14) transforms to the results of paper [8] in the limit $\mu \rightarrow \infty$. With the use of (14) we express $E$ as:

$$
\begin{array}{r}
E=\frac{20,25}{l^{2}+16 / \mu}-\frac{32 \alpha}{\sqrt{\pi}} \frac{1}{\sqrt{l^{2}+8 / \mu}} \\
+\frac{16 \alpha}{\sqrt{\pi}} \frac{1}{\sqrt{l^{2}+16 / \mu}}+\frac{6}{l^{2}} \\
+\frac{4 \sqrt{2}}{\sqrt{\pi}} \frac{\alpha}{1-\eta} \frac{1}{l},
\end{array}
$$

where $\eta=\varepsilon_{\infty} / \varepsilon_{0} \alpha=\left(e^{2} / \hbar \tilde{\varepsilon}\right) \sqrt{m / 2 \hbar \omega}$ - is a constant of electron-phonon coupling.

Let us write down $E_{\min }(\eta)$ for the minimum of the bipolaron total energy (15) with respect to the parameters $\mu$ and l. Fig. (1) shows the dependence of $E_{\text {min }}(\eta)$ (fig. 2 demonstrates the dependencies of $l_{\min }, \mu_{\min }$ on $\eta$ ) on the parameter $\eta$ in the range of its variation $(0,1)$. Fig. 1 suggests that $E_{\text {min }}(0)=-0,414125 \alpha^{2}$. Accordingly, for the critical value of the ion coupling parameter at which the bipolaron state is impossible it is equal to $\eta_{k p}=0,317, E_{\min }\left(\eta_{k p}\right)=2 E_{p}$ where $E_{p}=-0,1085128 \alpha^{2}$ is the polaron energy [11]. In [8] for $\eta_{k p}$, was found to be: $\eta_{k p}=0,2496$. The results obtained lead us to a considerably lower threshold of the formation of bipolaron states with respect to the electron-phonon interaction constant: $\alpha_{c} \geq 4,829$ which significantly expands the range of crystals in which bipolarons are possible. Previously the lowest value of $\alpha_{c}=6,8$ was obtained in [4].

Notice that $E_{\text {min }}$ determined by expression (14) yields much lower values of the bipolaron energy than all the results obtained earlier for all the values of $\eta$, and, in particular, the 


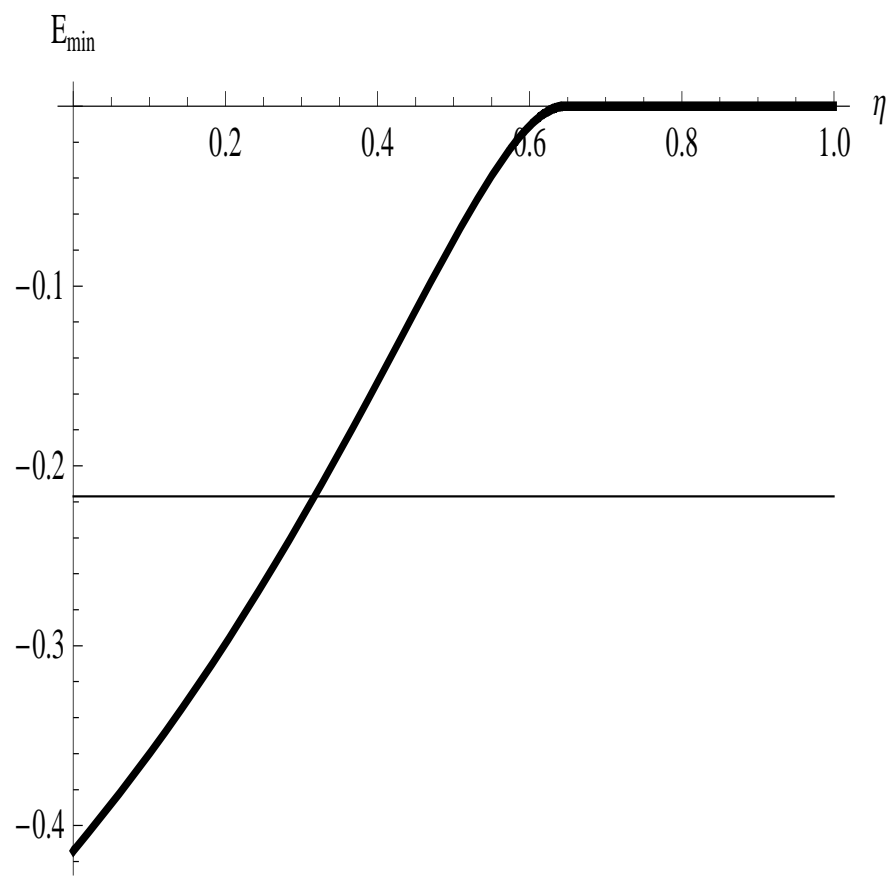

Figure 1: Function $E_{\min }(\eta)=\min _{x, y} E(x, y, \eta)$; a line $E=-0.217 ; E_{\min }(0)=$ -0.414125 .

value of $E_{\min }(0)=-0,3243 \alpha^{2}\left(\eta_{k p}=0,2496\right)$ found in [8] and $E_{\text {min }}(0)=-0,243628 \alpha^{2}-4,22606+0\left(\alpha^{-2}\right)$ derived in [12].

According to (14), the characteristic size of a bipolaron state is equal to $l$ and in dimensional units is: $l=\hbar^{2} \tilde{\varepsilon} x / m e^{2}$, where the dependence $x(\eta)$ is determined by that given in fig.2. From fig. 2 follows that on the whole interval of $\eta$ variation in the range of bipolaron states stability $\eta \in\left(0 ; \eta_{c}\right)$, the value $x$ changes only slightly from $x(\eta=0) \approx 6,3$ to $x(\eta=0,317) \approx 9,2$. Hence, even for $\eta=\eta_{c}$, the critical concentration of bipolarons at which their composite character makes itself evident, is $\eta_{c} \cong 10^{21} \mathrm{~cm}^{-3}$. If we take the maximum value of $\eta_{c}$ for a high-temperature superconductor $\mathrm{La}_{2} \mathrm{CuO}$ to be equal to $\eta_{c}=\eta_{c \perp}=0,174$ (the value of $\eta$ in the direction perpendicular to the $\mathrm{CuO}$ layers [13]), then the critical value of the concentration will be an order of magnitude greater than $\eta_{c}=10^{21} \mathrm{~cm}^{-3}$. These results provide support for the conclusion that 3D bipolaron mechanism of superconductivity is possible in copper oxides.

Being delocalized, these bipolaron states are translationinvariant (TI) and for $P=0$, where $P$ is the total momentum of the system, are separated by an energy gap from bipolaron states with spontaneously broken translation symmetry described by localized wave function [2]. For $P \neq 0$, TI bipolarons are not scattered on optical (acoustical) oscillations of the lattice [10], besides, as delocalized, they are not scattered on local defects of the lattice either. As is known [14, 15, 16], explanation of high-temperature superconductivity by Boseconcentration of bipolarons faces some problems associated with a large mass of these formations and, as a consequence, low values of $T_{c}$. The above indicated features of translation invariant bipolarons confer them superconducting properties even

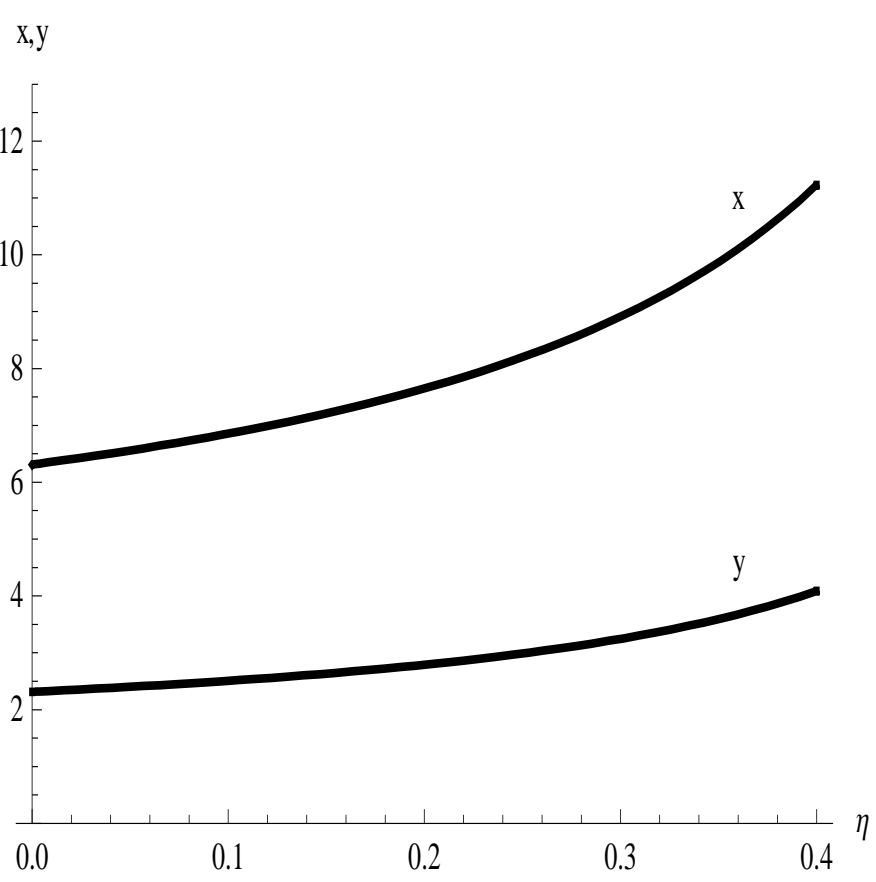

Figure 2: The dependence of $x=x_{\min }(\eta), y=y_{\min }(\eta)$ on $\eta ; x_{\min }(\eta), y_{\min }(\eta)$ correspond to minimum of $E(x . y ; \eta), x=\alpha l, y=\alpha^{2} / \mu$.

if their Bose-condensation is absent.

The work was supported by RFBR projects N 11-07-12054; 10-07-00112.

\section{References}

[1] A. S. Alexandrov, A. B. Krebs, Sov. Phys. Usp. 35 (1992) 345.

[2] N. I. Kashirina, V. D. Lakhno, Physics-Uspekhi 53 (5) (2010) 431-453.

[3] J. Adamowski, S. Bednarek, J. Phys.:Condensed Matter 4 (1992) 2845.

[4] G. Verbist, F. M. Peeters, J. T. Devreese, Phys. Rev. B 43 (1991) 2712.

[5] N. I. Kashirina, V. D. Lakhno, V. V. Sychyov, Physica status solidi (b) 234 (2002) 235.

[6] S. G. Suprun, B. Y. Moyzhes, Fiz. Tverd. Tela 24 (1982) 1580.

[7] N. I. Kashirina, V. D. Lakhno, V. V. Sychyov, Physica status solidi (b) 239 (2003) 174.

[8] V. D. Lakhno, JETP 137 (2010) 926.

[9] T. D. Lee, F. Low, D. Pines, Phys. Rev. 88 (1952) 960.

[10] A. V. Tulub, JETP 14 (1961) 1828.

[11] S. J. Miyake, J. Phys. Soc. Jap. 47 (1976) 747.

[12] M. A. Smondyrev, J. T. Devreese, F. M. Peeters, Phys. Rev. B 51 (1995) 15008.

[13] D. Reagor, E. Ahrens, S. W. Cheong, A. Migliori, Z. Fisk, Phys. Rev. Lett. 62 (1989) 2048.

[14] E. V. L. de Mello, J. Ranninger, Phys. Rev. B 55 (1997) 14872.

[15] Y. A. Firsov, V. V. Kabanov, E. K. Kudinov, A. S. Alexandrov, Phys. Rev. B 59 (1999) 12132.

[16] E. V. L. de Mello, J. Ranninger, Phys. Rev. B 59 (1999) 12135. 